\title{
Induced oral mucositis in Wistar rats treated with different drugs: Preventive potential in cytokine production
}

\author{
MARIA INÊS DA CRUZ CAMPOS ${ }^{1}$, CELSO NEIVA CAMPOS ${ }^{2}$, JOSÉ OTÁVIO AMARAL CORRÊA ${ }^{3}$, \\ FERNANDO MONTEIRO AARESTRUP ${ }^{4}$ and BEATRIZ JULIÃO VIEIRA AARESTRUP ${ }^{1}$
}

\begin{abstract}
${ }^{1}$ Laboratory of Immunopathology and Experimental Pathology, Reproductive Biology Center, Department of Morphology, Institute of Biological Sciences, Federal University of Juiz de Fora; ${ }^{2}$ School of Dentistry Clinic, Department of Dental Clinic, ${ }^{3}$ Laboratory of Pharmacology, Department of Immunohistochemistry, Federal University of Juiz de Fora; ${ }^{4}$ Laboratory of Immunopathology and Experimental Pathology, Reproductive Biology Center, Department of Dental Clinic, Faculty of Medical Sciences and Juiz de Fora Health-SUPREMA, Juiz de Fora, Minas Gerais 36036-330, Brazil
\end{abstract}

Received July 11, 2019; Accepted April 1, 2021

DOI: $10.3892 / \mathrm{mco} .2021 .2289$

\begin{abstract}
The aim of the present study was to investigate the preventive potential of pentoxifylline, atorvastatin and trans-caryophyllene in oral mucositis through histopathological analysis of wounds in the oral mucosa of Wistar rats treated with 5-FU, and to evaluate the immunomodulatory effect of these drugs on serum nitrite production, in situ IFN- $\gamma$, TNF- $\alpha$ and TGF- $\beta$, and TNF- $\alpha$ in tissues. A total of 32 male Wistar rats with an average age of 9 weeks and an average body weight of $250 \mathrm{~g}$ were divided into four treatment groups: Saline, trans-caryophyllene, pentoxifylline and atorvastatin. Oral mucositis was then induced. On days 3 and 4, the mucosa of the mouth of eight pre-treated animals in each group was bilaterally scarified twice with the tip of a sterile needle, with an anesthetic solution. Mucosal samples from animals treated with trans-caryophyllene preserved a thin epithelial lining associated with focal perivascular inflammatory infiltrates. Pentoxifylline-treated animals exhibited total epithelial loss in oral wounds with severe inflammatory infiltrates and mild re-epithelialization associated with mild and diffuse inflammatory infiltrates. Samples from atorvastatin-treated animals exhibited no epithelial dissolution, with preserved thin lining and mild diffuse inflammatory infiltrates. The analysis of TNF- $\alpha$ expression revealed improved results in trans-caryophyllene animals. The analysis of TGF- $\beta$ expression revealed positive mononuclear cells. Preventive treatment with atorvastatin was demonstrated to modulate the serum
\end{abstract}

Correspondence to: Professor Maria Inês da Cruz Campos, Laboratory of Immunopathology and Experimental Pathology, Reproductive Biology Center, Department of Morphology, Institute of Biological Sciences, Federal University of Juiz de Fora, Lindalva de Paula Ribeiro, 240 Bosque Imperial, Juiz de Fora, Minas Gerais 36036-330, Brazil

E-mail: marimurucci@gmail.com

Key words: leukopenia, oral mucositis, trans-caryophyllene, atorvastatin, chemotherapy expression levels of TNF- $\alpha$ during all stages of the experiment. Treatment with trans-caryophyllene modulated serum IFN- $\gamma$ levels negatively, whereas treatment with atorvastatin and trans-caryophyllene maintained lower levels of IFN- $\gamma$ compared with the control group.

\section{Introduction}

Oral mucositis affects $40-80 \%$ of patients undergoing chemotherapy and almost all patients undergoing head and neck radiotherapy (1-3). Oral mucositis causes dysphagia, dysarthria, and odynophagia and is a possible gateway for opportunistic infections; therefore, it leads to a decreased quality of life and is considered an important non-hematological complication of antitumor treatment (4-8).

Typical macroscopic and microscopic characteristics of oral mucositis reflect a natural history following radiation therapy or the chemotherapy cycle, in which the initial wound of an erythematous plaque evolves into isolated ulcerations that converge and result in more extensive and deeper wounds, with symptoms ranging from burning to severe pain (4). The erythematous wound histopathologically represents epithelial hypoplasia associated with inflammatory reaction on the underlying lamina propria caused by an antitumor drug that secondarily inhibits normal epithelial renewal; the healing phase occurs with the end of the antineoplastic cycle, showing spontaneous resolution of the ulcers (7,9-11).

The in situ cytokine production by oral inflammatory cells influences the establishment and remission of ulcers. Parkin and Cohen (12) suggested that the cytokine profile of the lamina propria cells affects the mitotic and apoptotic activities of oral epithelial tissue. Notable among these cytokines, are tumor necrosis factor-alpha (TNF- $\alpha$ ), transforming growth factor-beta (TGF- $\beta$ ), and interferon-gamma (IFN- $\gamma$ ). TNF- $\alpha$ is a pro-inflammatory cytokine that is primarily produced by activated macrophages. TGF- $\beta$ enhances the proliferation of several cells of mesenchymal origin and increases extracellular matrix synthesis by T-lymphocytes and platelets. IFN- $\gamma$ is produced by Th1 lymphocytes and natural killer cells; it regulates the 
proliferation and differentiation of various cell types and has the ability to modulate the immune system (13-15).

Besides cytokines, nitric oxide is one of the ten smallest molecules existing in nature and is a highly soluble reactive free radical produced by the NO synthase (NOS) enzyme from L-arginine. This molecule remains in the tissue for only a few seconds, but its presence can be indirectly detected through NOS based on its tissue expression namely eNOS (endothelial), nNOS (neuronal), and iNOS (inducible) (16-18). Experimental and clinical studies suggest that both overproduction and inhibition of NO are associated with greater potential for tissue damage or maintenance of a chronic response, respectively (17).

Nitric oxide also has a destructive effect on invading microorganisms and is therefore released into the inflamed site by neutrophils and macrophages (19).

Some immunomodulatory drugs have been employed to control the exacerbation of oral mucositis wounds (20-22). Among these, pentoxifylline (PTX) is an anti-bleeding and anti-thrombogenic agent with the ability to inhibit the TNF- $\alpha$ expression by inhibiting its genetic transcription $(23,24)$. Atorvastatin has an anti-inflammatory and anti-thrombogenic action that is capable of reducing $\mathrm{C}$-reactive protein levels and TNF- $\alpha$ expression in situ $(20,25)$.

Studies conducted over the past decade have also demonstrated the effectiveness of the anti-inflammatory action of trans-caryophyllene, a molecule isolated from the copaiba oil-resin of the Brazilian medicinal plant Copaifera langsdorffii (25-28).

Although the current control of oral wounds is non-specific and palliative-preventing their establishment is critical to cancer prognosis, since severe wounds limit and disrupt treatment $(4,6,29,30)$.

In our previous study, utilizing the same experimental design as the current study, we determined the influence of these drugs on white blood cell counts of animals undergoing chemotherapy with 5-fluorouracil (5-FU). The results demonstrated that atorvastatin significantly prevented leukocyte reduction in comparison to other experimental groups, thus demonstrating excellent potential for the prevention of leukopenia (25).

Considering the increase in the number of patients submitted to chemotherapy and/or radiotherapy treatments for cancer control and, consequently, sequelae caused by those treatments, this article discusses the need to control these manifestations with the objective of improving the quality of life of the aforementioned patients. The large number of cancer cases makes the use of such therapies to increase even further, as they are considered the only way to control the development of the disease. As a consequence of the use of these therapies, oral mucositis might appear, which leads to poor nutrition of the patient, difficulties to swallow and, generally, causes the abandonment of treatment due to lack of control. Given the fact that there is little knowledge on the control and prevention of such oral ulcers, new studies are necessary to unveil information that might be useful in such situations.

The aim of the present study was to investigate the preventive potential of pentoxifylline, atorvastatin and trans-caryophyllene based on histopathological analysis of wounds in the oral mucosa induced in 5-FU-treated Wistar rats, and to evaluate their immunomodulatory effect on serum nitrite production, in situ IFN- $\gamma$ and TNF- $\alpha$, and TGF- $\beta$ and TNF- $\alpha$ in tissues.

\section{Materials and methods}

Approval and separation of animals. The entire experimental protocol was approved by the Animal Research Committee of the Federal University of Juiz de Fora (Notion No. 062/2011).

Male Wistar rats (Rattus norvegicus) $(\mathrm{N}=32)$ from the Animal Facility of the Reproductive Biology Center (CBR) of the Federal University of Juiz de Fora were used for this study, with an average age of 9 weeks and average body weight of $250 \mathrm{~g}$. The rats were allowed ad libitum access to food and water.

Fifteen days before induction of oral mucositis, the animals were divided into four groups: Group I-saline-treated animals with chemotherapy-induced mucositis $(n=8)$, Grou p II-trans-caryophyllene-treated animals with chemotherapy-induced mucositis $(n=8)$, Group III-pentoxifylline- $t$ reated animals with chemotherapy-induced mucositis $(n=8)$, and Group IV-atorvastatin-treated animals with chemotherapy-induced mucositis $(n=8)$.

Drug delivery protocol. Trans-caryophyllene gavage administration began two days before 5-FU treatment and was continued for 2 days after medication (totalling 9 days of treatment) with a dose of $50 \mathrm{mg} / \mathrm{kg}(0.102 \mathrm{ml})(31-33)$.

Pentoxifylline dissolved in sterile saline of $0.9 \%$ at a concentration of $100 \mathrm{mg} / \mathrm{kg} /$ day that was administered intraperitoneally for 15 consecutive days (34).

Atorvastatin was administered intraperitoneally at $10 \mathrm{mg} / \mathrm{kg} /$ day for 1 week before 5 -FU treatment (20).

The drug delivery schedule is outlined in Fig. 1.

Induction of oral mucositis. Oral mucositis was induced by the administration of the chemotherapeutic agent 5-FU (Eurofarma, São Paulo, Brazil) via intraperitoneal injection on Day 0 $(100 \mathrm{mg} / \mathrm{kg})$ and on Day $2(60 \mathrm{mg} / \mathrm{kg})$ of the experiment (35).

On Days 3 and 4, the mouth's mucosa of 8 pre-treated animals from each group, after being anesthetized with Ketamine $(100 \mathrm{mg} / \mathrm{kg})$ and Xylazine $(10 \mathrm{mg} / \mathrm{kg})$, was bilaterally scarified twice by the same operator using the tip of a sterile needle $(35,36)$.

Determination of when the animals should be sacrificed. The animals were separated into groups to be euthanized at 8,11 and 15 days after induction of oral mucositis. However, clinical evaluation standards were used that could cause the anticipation of deaths: Marked weight loss, ocular or nasal hemorrhage, decreased consumption of water and feed, loss of movement in the cage, demonstration of pain.

Euthanasia method. The method used was deep anestesia with the combination of Xylazine $10 \mathrm{mg} / \mathrm{kg}+100 \mathrm{mg} / \mathrm{kg}$ Ketamine, mixed in the same syringe, intraperitoneally.

The death of the animals was confirmed through cardiac and respiratory arrest, absence of reflexes (seen through the hind legs), drop in body temperature.

After the drug is applied, exsanguination is performed, performed by means of cardiac puncture or large blood vessels, when rodent serum is obtained. 


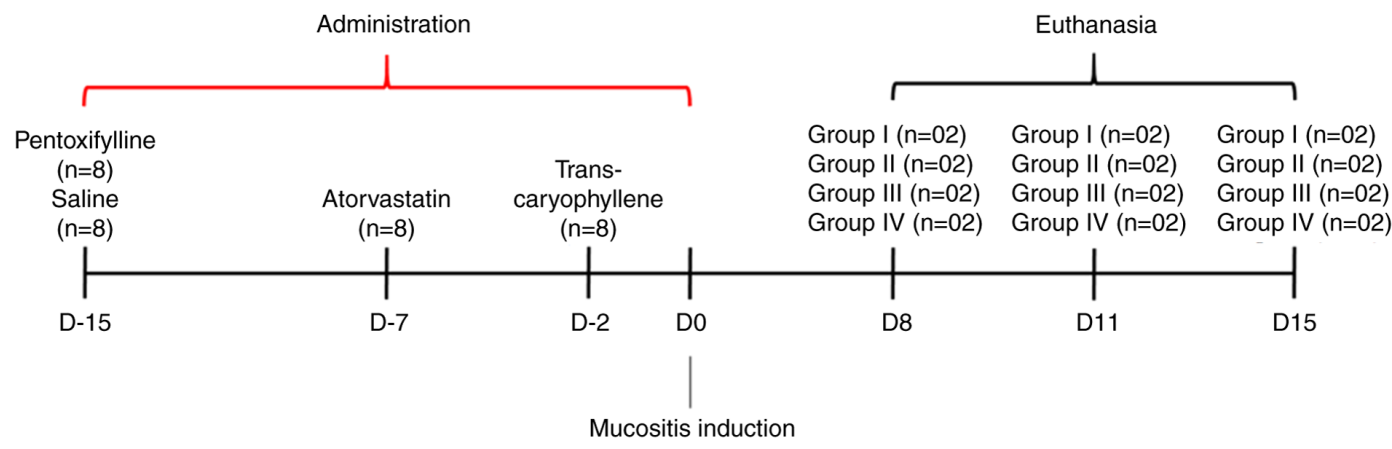

Figure 1. Drug delivery schedule by group of animals and days of euthanasia. Day 0: Day of mucositis induction; Day 15: Initial preventive treatment; Days 8, 11, and 15: Days of euthanasia with oral mucosa and serum sampling. Group V includes two animals from each drug group without mucositis. D, day.

Sample collection and analysis. After deep anesthesia with Ketamine $(100 \mathrm{mg} / \mathrm{kg})$ and Xylazine $(10 \mathrm{mg} / \mathrm{kg})$, via intraperitoneal injection, followed by euthanasia, the mouth's mucosa was excised.

The schedule of euthanasia, as well as the drug delivery, is outlined in Fig. 1.

The excised mouth's mucosa was dissected to obtain the mucositis-affected areas. The samples were immediately fixed in $10 \%$ buffered formalin and submitted to routine histological processing for $\mathrm{H} \& \mathrm{E}$ staining.

The sections were analyzed with a x400 magnification Zeiss microscope (Hallbergmoos) in three fields of each sample by an Axion Cam ICC 5 (Zeiss) computer system digital capture performed by means of a digital camera attached to an optical microscope. The captured images were processed in an automatic morphometry Zen 2012 (Blue Edition) program, in which semi-automatic microscopic field morphometry of leukocytes and vascular sections was performed on the lamina propria underlying the epithelial wound.

Body weight. Animals should not lose more than $30 \%$ of their initial body weight. For this, both body weight and feed intake should were evaluated daily. As the animals were controlled daily, when there was excessive weight loss, they were euthanized.

In situ detection of TNF- $\alpha$ and TGF- $\beta$ expression. The Starr Trek Universal HRP Detection System was used to analyze the expression of TGF- $\beta$ and TNF- $\alpha$ in the lamina propria cells that were considered positive by intracytoplasmic brown pigmentation. Three field counts were averaged, in addition to total counts for each group.

TNF- $\alpha$ and IFN- $\gamma$ serum levels. TNF- $\alpha$ and IFN- $\gamma$ (both from PeproTech Inc.) levels were examined by the ELISA (Enzyme Linked ImmunoSorbent Assay) method, which is based on antigen-antibody interactions detected through enzymatic reactions. In this method, an antigen is bound to a solid base-the ELISA plate- and is subsequently tested. In the case of a positive result, an antigen-antibody binding occurs, which is detected by adding a secondary antibody that targets the immunoglobulins of the target species (37).

Serum nitrite levels. Serum nitrite was quantified using the Griess method, by generating a standard curve covering

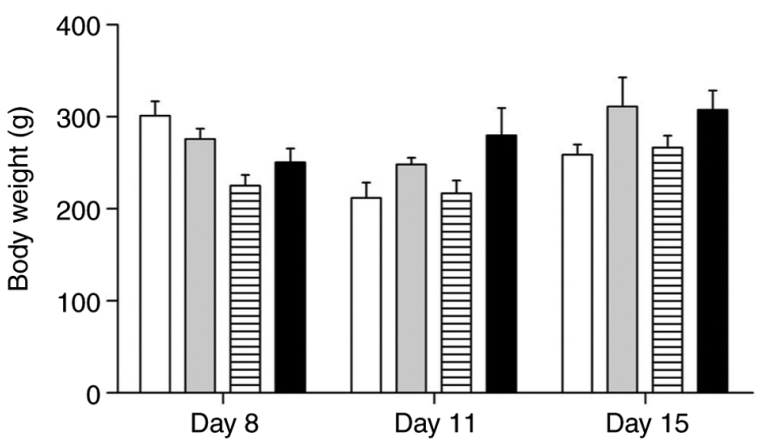

Figure 2. Body weight variation in animals upon euthanasia on different days of the experiment. Group I animals exhibited marked weight loss of 50\% on the 11th day and slight recovery at the end of the experiment. Group II animals showed slight initial weight loss and recovery $>50 \%$ by the end. Group III showed weight loss leading to cachexia on the 11th day and slight recovery until the 15 th day. Group IV animals showed a weight gain $>50 \%$ on all days of the experiment. White column, saline; grey column, trans-cariophyllene; horizontal lines column, pentoxifylline; black column, atorvastatin.

concentrations between 3.12 and $100 \mu \mathrm{M}$. The results were expressed in moles $/ \mathrm{ml}$. The Griess method or test is a chemical reaction that detects the presence of organic nitrites through its reaction with sulfanilamide in an acidic medium. To measure nitrite production, $100 \mu 1$ aliquots of samples were incubated with $100 \mu \mathrm{l}$ of the Griess reagent $(50 \mu 11 \%$ sulfanilamide solution and $50 \mu 10.1 \% \mathrm{~N}$-naphthyl ethylenediamine dihydrochloride solution in $2.5 \% \mathrm{H}_{2} \mathrm{PO}_{4}$ ) at room temperature for $10 \mathrm{~min}$. This reaction produces a bright red staining compound that can be estimated from $10 \mathrm{~min}$ to $2 \mathrm{~h}$ after mixing (38).

Statistical analysis. The data are presented as mean \pm standard deviation of the mean (SEM). For comparison between groups, we used a Kruskal-Wallis test with Dunn's Multiple Comparison post-hoc test. In all instances, the significance level was set at $5 \%(\mathrm{P}<0.05)$. The analyses were performed with GraphPad Prism version 5.0 for Windows (GraphPad Software).

\section{Results}

Body weight. Although all animals showed weight loss, there was a significant variation between different groups. The results were expressed as the mean body weight per group on different days of the experiment (Fig. 2). 


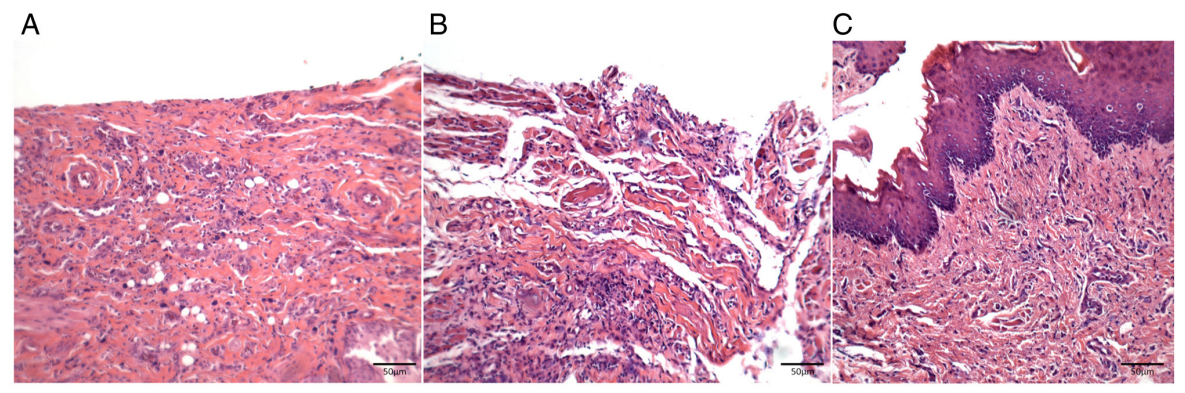

Figure 3. Photomicrographs of histological sections of oral mucosa samples from Wistar rats subjected to chemotherapy. (A) Control group (day 8), with diffuse inflammatory infiltrate and loss of epithelial continuity. (B) Control group (day 11), showing that oral wounds progressed to ulcers with severe diffuse inflammatory infiltrate. (C) Control group (day 15) with mild re-epithelialization associated with underlying inflammatory infiltrate still present. H\&E staining. Magnification, x100.

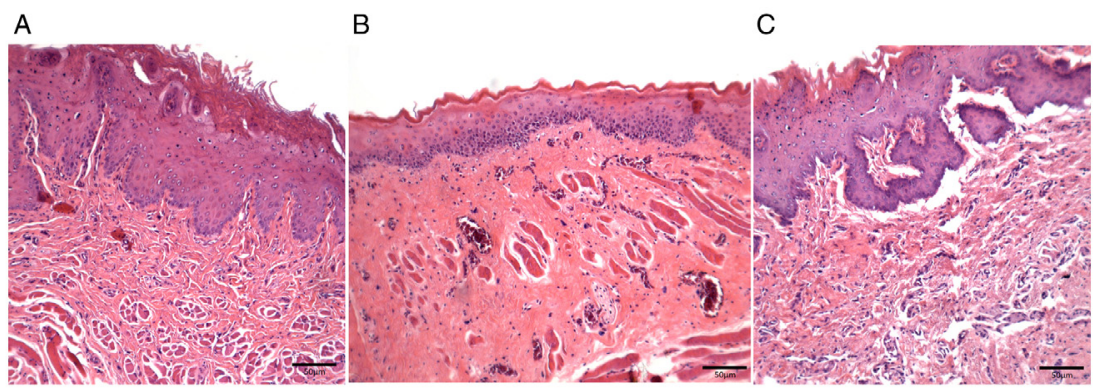

Figure 4. Photomicrographs of histological sections of oral mucosa samples from Wistar rats subjected to chemotherapy. (A) Trans-caryophyllene group (day 8), showing preserved thin epithelial lining associated with focal perivascular inflammatory infiltrate. Areas were occasionally identified with no leukocyte infiltration. (B) Trans-caryophyllene group (day 11), no inflammation in the samples and decreased amount of vascular sections. (C) Trans-caryophyllene group (day 15), with keratinized epithelial restoration and recovery of the number of vascular sections. H\&E staining. Magnification, x100.

Group I animals showed marked weight loss of around $50 \%$ on the 11th day and slight recovery at the end of the experiment; Group II animals showed slight initial weight loss and recovery greater than $50 \%$ by the end of it; Group III showed weight loss leading to cachexia on the 11th day and slight recovery until the 15th day; Group IV animals showed a weight gain greater than $50 \%$ on all days of the experiment.

Histopathological and histomorphometric analysis. At 8 days after delivery of the chemotherapeutic drug, samples from the saline-treated control group of animals (Group I) showed loss of epithelium in the oral mucosa with mild inflammatory mononuclear infiltrates. At 11 days after chemotherapy in these animals, the oral wounds progressed to ulcers with severe diffuse inflammatory infiltrates and at 15 days after chemotherapy, the wounds showed mild re-epithelialization associated with an underlying inflammatory infiltrate still present (Fig. 3A-C).

Mucosal samples in trans-caryophyllene-treated animals (Group II) showed a preserved thin epithelial lining associated with focal perivascular inflammatory infiltrates on Day 8 and areas with no leukocyte infiltration were occasionally identified; no inflammation was observed in the samples on Day 11, and on Day 15 keratinized epithelial restoration was observed. Moreover, the amount of vascular sections decreased on Day 11 and showed recovery on Day 15 (Fig. 4A-C).

From Day 8 onwards, samples from the pentoxifylline-treated animals (Group III) showed early and complete epithelial loss in oral wounds with severe inflammatory infiltrates and occasional necrotic areas; on Day 15 slight re-epithelialization associated with a mild and diffuse inflammatory infiltrate was observed. The number of blood vessels decreased in animals from this group on Day 11 before it increased again on Day 15 (Fig. 5A-C).

On Day 8, samples from atorvastatin-treated animals (Group IV) displayed no epithelial dissolution, with preserved thin lining and mild diffuse inflammatory infiltrates; on Days 11 and 15 there was a progressive increase in inflammatory infiltrates, but with epithelial preservation. Similar to other groups, recovery of the number of vascular sections was observed on Day 15, after a decrease on Day 11 (Fig. 6A-C).

The morphometric analysis of the inflammatory infiltrates and the wound area vascularization is shown in Figs. 7 and 8.

In situ analysis of TNF- $\alpha$ and TGF- $\beta$ expression. The analysis of TNF- $\alpha$ expression revealed many positive mononuclear cells, mainly in samples from trans-caryophyllene-treated animals (Fig. 9). Atorvastatin negatively modulated the levels of this cytokine during mucositis development at all stages $(\mathrm{P}<0.01)$. Pentoxifylline-treated animals maintained a number of positive cells similar to the control group (Fig. 10).

Similarly, the analysis of TGF- $\beta$ expression in the lamina propria infiltrating inflammatory cells contained in oral mucosa samples revealed several positive mononuclear cells, observed mainly in samples from atorvastatin-treated animals (Fig. 11). The lowest number of cells was observed in the group treated with trans-caryophyllene $(\mathrm{P}<0.01)$ (Fig. 12). 


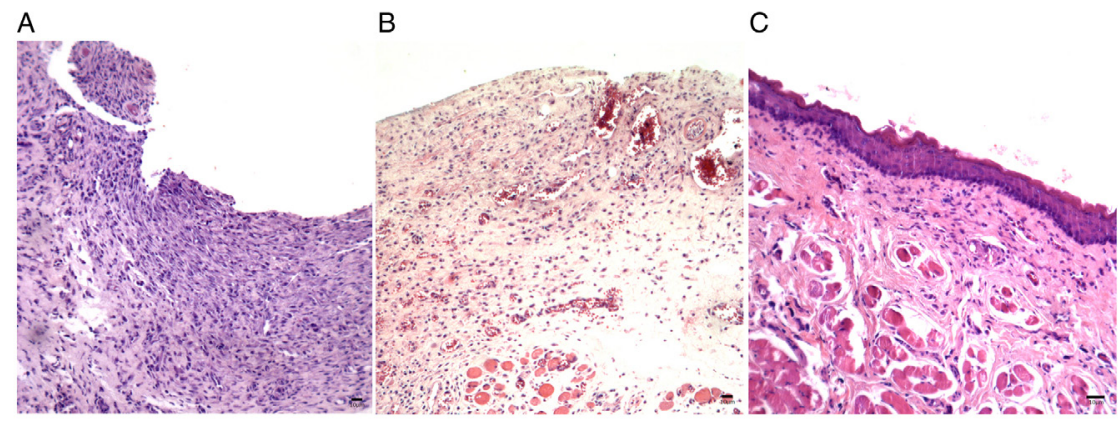

Figure 5. Photomicrographs of histological sections of oral mucosa samples from Wistar rats subjected to chemotherapy. (A) Pentoxifylline group (day 8) with total epithelial loss of oral wounds with severe inflammatory infiltrate. Necrotic areas were occasionally observed. (B) Pentoxifylline group (day 11) continued total epithelial loss of oral wounds with severe inflammatory infiltrate and decreased number of blood vessels. (C) Pentoxifylline group (day 15), mild re-epithelialization associated with mild and diffuse inflammatory infiltrate. Increased vascular sections. H\&E staining. Magnification, x100.
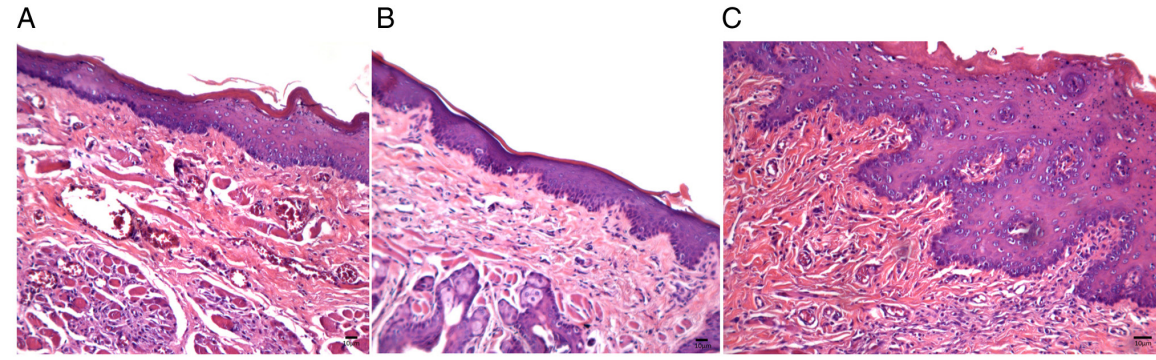

Figure 6. Photomicrographs of histological sections of oral mucosa samples from Wistar rats subjected to chemotherapy. (A) Atorvastatin group (day 8), no epithelial dissolution, with preserved thin lining and mild diffuse inflammatory infiltrate. (B) Atorvastatin group (day 11), with progressive increase of inflammatory infiltrate, but with epithelial preservation and reduced number of vascular sections. (C) Atorvastatin group (day 15), with recovered number of vascular sections and increased epithelial thickness. H\&E staining. Magnification, x100.

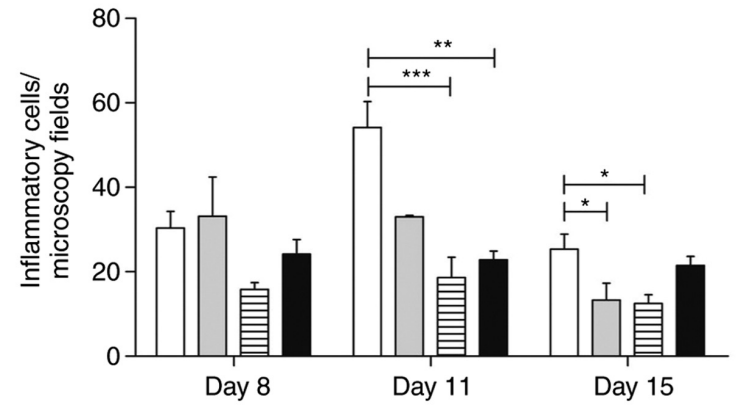

Figure 7. Number of inflammatory cells per group on different days of the experiment. White column, saline; grey column, trans-cariophyllene; horizontal lines column, pentoxifylline; black column, atorvastatin. ${ }^{~} \mathrm{P}<0.05$; ${ }^{* *} \mathrm{P}<0.01 ;{ }^{* * *} \mathrm{P}<0.001$.

Serological evaluation of TNF- $\alpha$ and INF- $\gamma$. Preventive treatment with atorvastatin has been shown to modulate TNF- $\alpha$ levels in serum samples during all stages evaluated in the experiment $(\mathrm{P}<0.01)(277.3 \pm 45.12$ on Day $8 ; 285.3 \pm 30.63$ on Day $11 ; 200.3 \pm 12.20$ on Day 15$)$ in relation to the samples from the control group (194.0 \pm 30.19 on Day 8; 303.0 \pm 25.60 on Day 11; and 286.50 \pm 23.10 on Day 15) (Fig. 13).

On the other hand, the trans-caryophyllene treatment negatively modulated the serum levels of IFN- $\gamma$ on the first day of analysis $(\mathrm{P}<0.05)(117.8 \pm 28.91)$, compared to the control group (194.0 \pm 30.19$)$, whereas atorvastatin delivery was effective in modulating this cytokine on Day 11 $(99.00 \pm 12.25)$. On Day 15, the treatments with atorvastatin

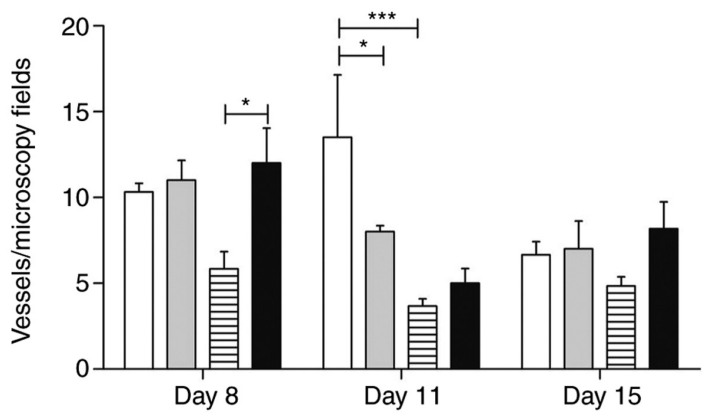

Figure 8. Number of vascular sections per group on different days of the experiment. White column, saline; grey column, trans-cariophyllene; horizontal lines column, pentoxifylline; black column, atorvastatin. ${ }^{*} \mathrm{P}<0.05$; ${ }^{* * *} \mathrm{P}<0.001$

and trans-caryophyllene maintained significantly lower IFN $-\gamma$ levels than those of the control group $(75.00 \pm 19.27$, and $67.50 \pm 4.65$, respectively). Pentoxifylline treatment did not influence the IFN- $\gamma$ serum levels in the animals at any stage evaluated in the study. Details of the IFN- $\gamma$ serum levels are shown in Fig. 14.

Serological nitrite evaluation. The serum nitrite levels were significantly modulated by trans-caryophyllene on all evaluated days $(\mathrm{P}<0.05)(8.12 \pm 1.8 ; 13.97 \pm 2.69 ; 17.28 \pm 1.75)$; atorvastatin, in turn, was effective only in the last period $(22.8 \pm 3.25$, Day 15), compared to the control group on the same days. Pentoxifylline treatment did not influence the serum nitrite 
A

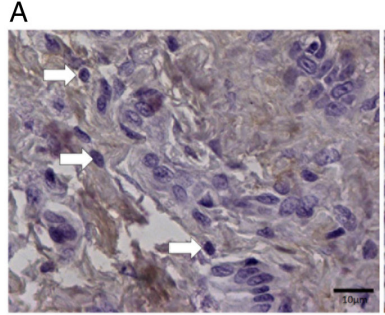

B

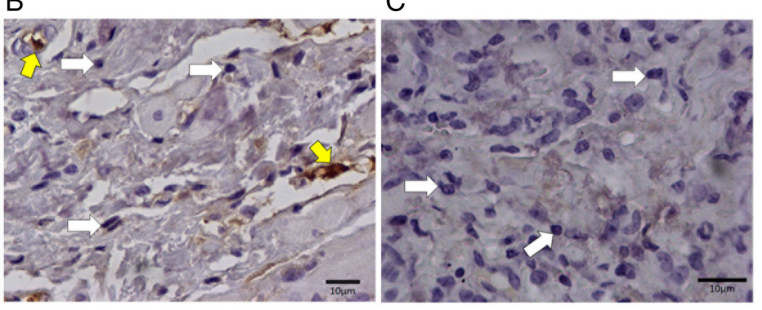

D
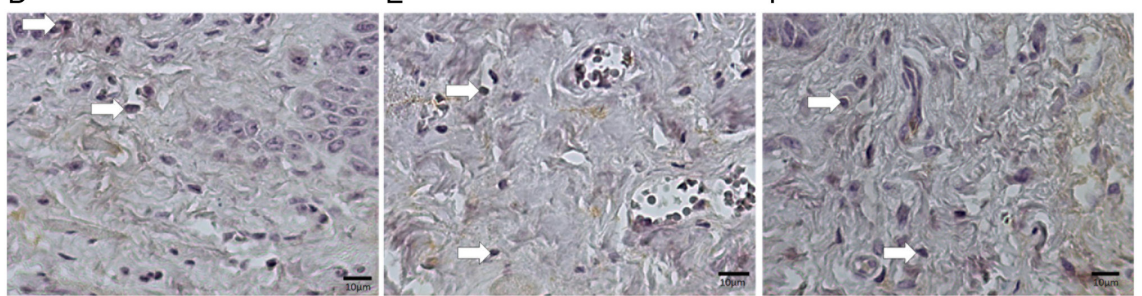

G

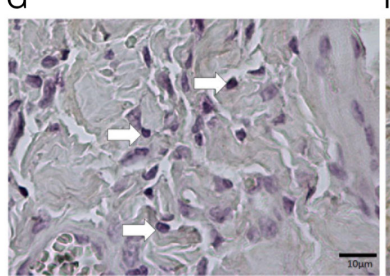

$\mathrm{H}$

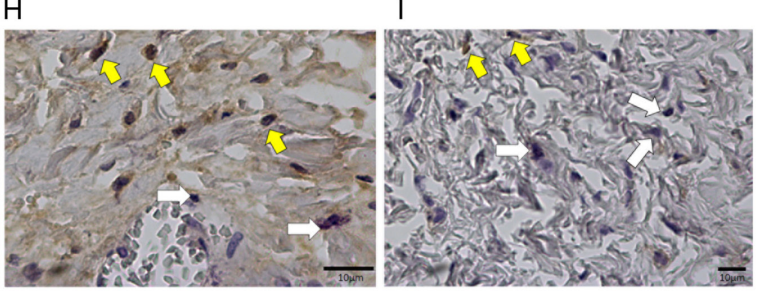

$J$

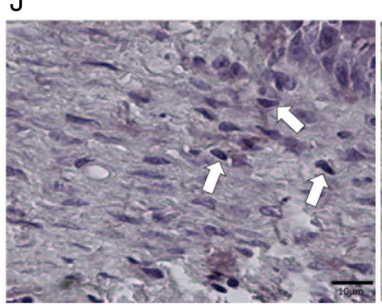

$\mathrm{K}$

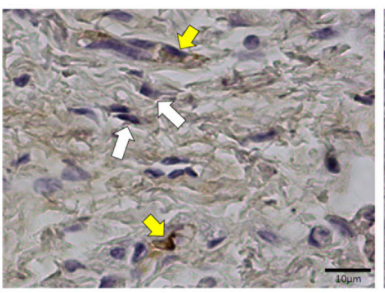

L

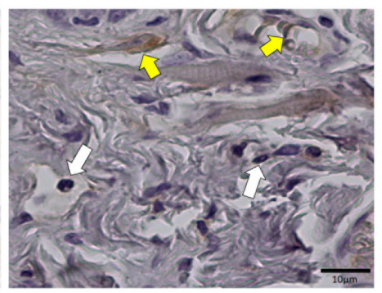

Figure 9. Photomicrographs of histological sections of oral mucosa samples from Wistar rats subjected to chemotherapy. (A) Animal in the control group, without medication, euthanized on day 8. (B) Animal in the control group, without medication, euthanized on day 11. (C) Animal in the control group, without medication, euthanized on the 15th day. (D) Animal that received trans-karyophylene, euthanized on day 8. (E) Animal that received euthanized trans-karyophylene on day 11. (F) Animal that received trans-karyophylene, euthanized on the 15th day. (G) Animal that received pentoxifylline, euthanized on day 8. (H) Animal that received pentoxifylline, euthanized on day 11. (I) Animal that received pentoxifylline, euthanized on day 15. (J) Animal that received atorvastatin, euthanized on the 8th day. (K) Animal that received atorvastatin, euthanized on the 11th day. (L) Animal that received atorvastatin, euthanized on day 15. Magnification, x400. White arrows show negative cells and yellow arrows indicate positive cells. Anti-TNF- $\alpha$ immunohistochemical reaction.

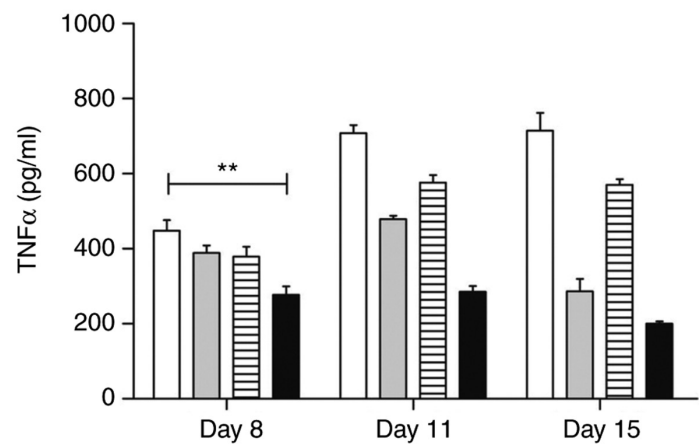

Figure 10. Comparison of TNF- $\alpha$ levels on different days of the experiment. White column, saline; grey column, trans-cariophyllene; horizontal lines column, pentoxifylline; black column, atorvastatin. ${ }^{* *} \mathrm{P}<0.01$

levels in the different evaluation periods. Fig. 15 shows the serum nitrite levels in different groups of animals throughout the study.

\section{Discussion}

Oral mucositis has been the focus of several experimental and clinical studies because its control allows more effective anti-cancer radiation therapy and/or chemotherapy. However, despite its importance, no control or preventive treatment for oral mucositis has been established or filed so far (6,7,9,39-44). To this end, several experimental models have been developed to investigate the mechanisms associated with the oral mucositis development, and to assess the effects of different therapeutic agents on the evolution of oral ulcers $(1,8,9,20,22,30,35,45)$.

In this study, histopathological and histomorphometric analyses of oral mucosa samples demonstrated that treatment with atorvastatin and pentoxifylline proved effective in modulating the cellularity of the inflammatory infiltrate on Day 11 of the experiment, corresponding to the exacerbation of oral mucositis. Trans-caryophyllene, as well as pentoxifylline, showed significant effects on Day 15, in the remission stage of oral ulcers. The delivery of pentoxifylline 

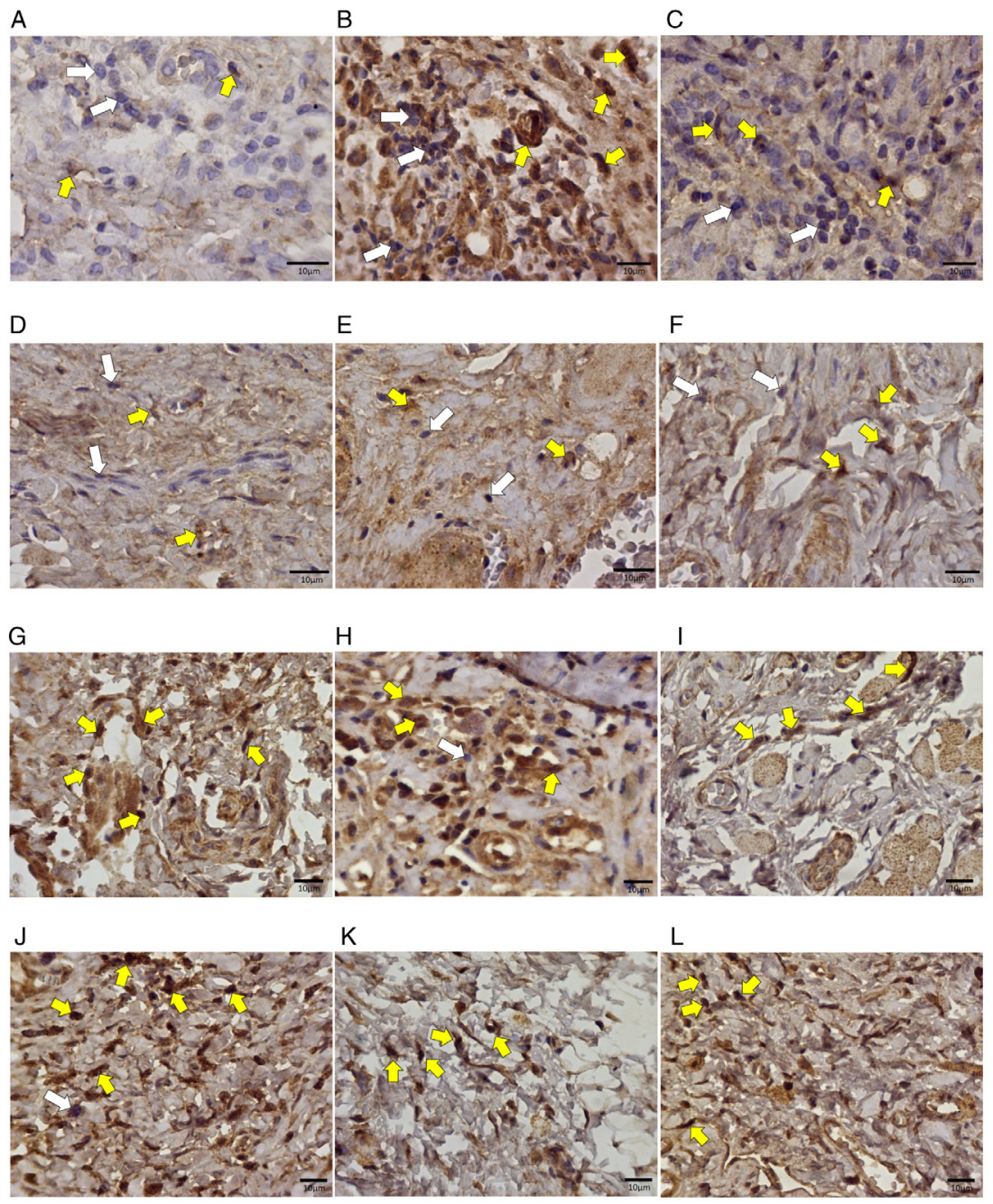

Figure 11. Photomicrographs of histological sections of oral mucosa samples from Wistar rats subjected to chemotherapy. (A) Animal in the control group, without medication, euthanized on day 8. (B) Animal in the control group, without medication, euthanized on day 11. (C) Animal in the control group, without medication, euthanized on the 15th day. (D) Animal that received trans-karyophylene, euthanized on day 8. (E) Animal that received euthanized trans-karyophylene on day 11. (F) Animal that received trans-karyophylene, euthanized on the 15th day. (G) Animal that received pentoxifylline, euthanized on day 8. (H) Animal that received pentoxifylline, euthanized on day 11. (I) Animal that received pentoxifylline, euthanized on day 15. (J) Animal that received atorvastatin, euthanized on the 8th day. (K) Animal that received atorvastatin, euthanized on the 11th day. (L) Animal that received atorvastatin, euthanized on day 15. Magnification, $\mathrm{x} 400$. White arrows indicate negative cells and yellow arrows indicate positive cells for the anti-TGF- $\beta$ immunohistochemical reaction.

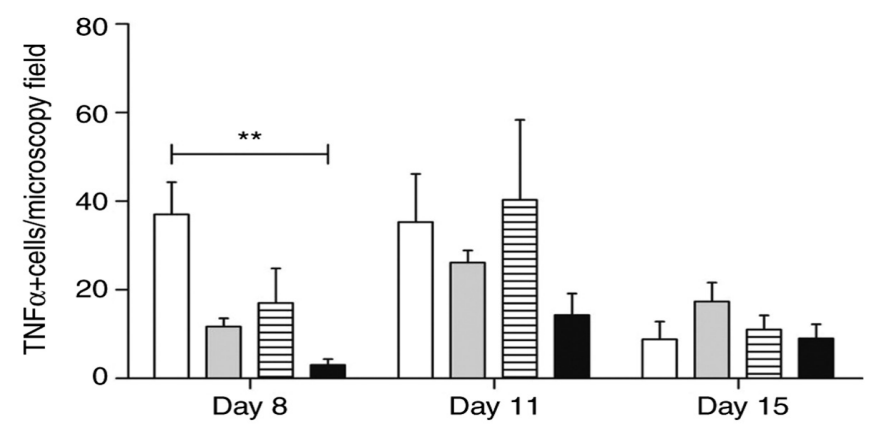

Figure 12. Comparison of TNF- $\alpha$ levels on different days of the experiment. White column, saline; grey column, trans-cariophyllene; horizontal lines column, pentoxifylline; black column, atorvastatin. ${ }^{* *} \mathrm{P}<0.01$.

and trans-caryophyllene favored angiogenesis on the lamina propria of Day 11 samples. These data suggest that there was no correlation between the severity of the inflammatory infiltrate and the amount of vascular sections analyzed per

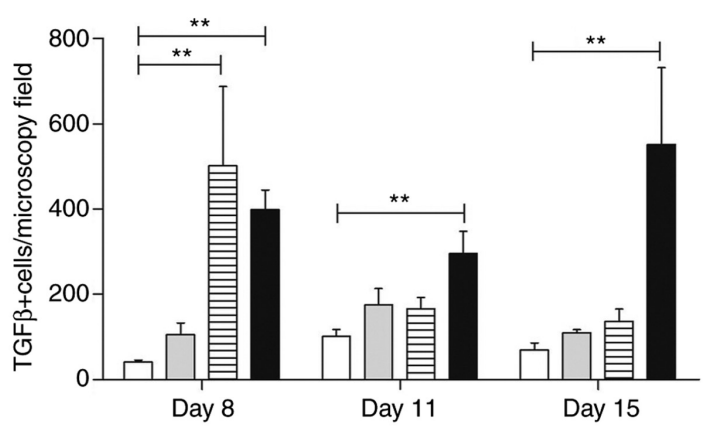

Figure 13. Comparison of TGF- $\beta$ levels on different days of the experiment. White column, saline; grey column, trans-cariophyllene; horizontal lines column, pentoxifylline; black column, atorvastatin. ${ }^{* *} \mathrm{P}<0.01$.

microscopic field. However, the local inflammatory infiltrate influenced the loss of epithelial continuity. Lima et al (46) showed the development of local ulcers and abscesses associated with leukopenia and a change in body mass in a hamster 


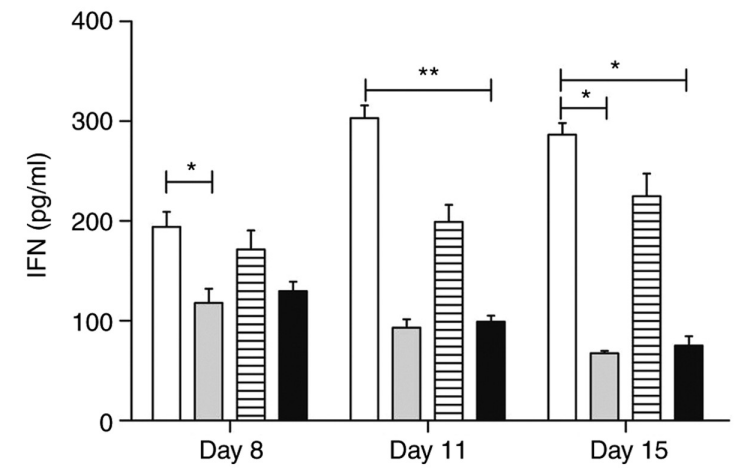

Figure 14. Comparison of IFN- $\gamma$ levels on different days of the experiment. White column, saline; grey column, trans-cariophyllene; horizontal lines column, pentoxifylline; black column, atorvastatin. ${ }^{*} \mathrm{P}<0.05 ;{ }^{* * *} \mathrm{P}<0.01$.

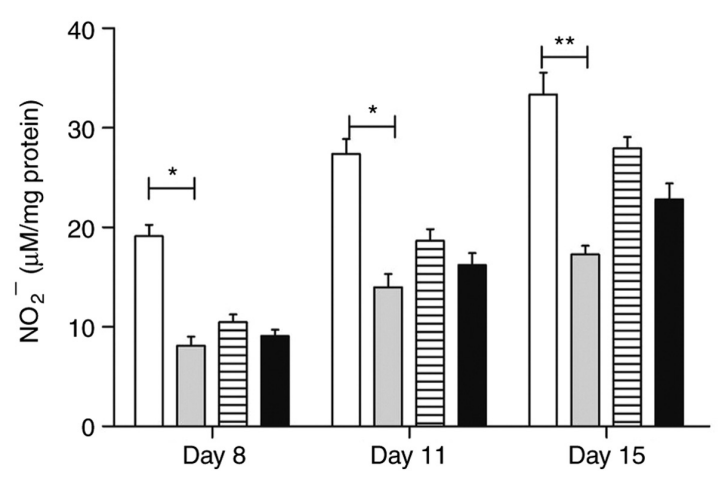

Figure 15. Comparison of serum nitrite levels on different days of the experiment. White column, saline; grey column, trans-cariophyllene; horizontal lines column, pentoxifylline; black column, atorvastatin. ${ }^{*} \mathrm{P}<0.05 ;{ }^{* *} \mathrm{P}<0.01$.

model. Jain et al (47) used the oral mucositis model in rats to prove that complex biological events mediated by a number of inflammatory cytokines and their direct effect on the basal layer of the epithelium result in the destruction of the anatomical barrier of the oral mucosa.

Results from studies by Ward and Clissold (34) and Allen et al (48) suggested that controlling the TNF- $\alpha$ expression by inhibiting its gene transcription allows it to modulate leukopenia and inflammatory infiltrates in the mucosa affected by oral mucositis (23). This study showed that the TNF- $\alpha$ serum levels were significantly lower in samples that were treated preventively with atorvastatin compared to the control group and other experimental groups. The in situ expression of this cytokine was lower in the trans-caryophyllene-treated group and was histopathologically associated with lower inflammation of the oral ulcers and their satisfactory re-epithelialization. These results suggest that preventive therapy with atorvastatin is capable of controlling ulceration of the oral mucosa by inhibiting TNF- $\alpha$ production.

Preventive treatment with trans-caryophyllene has been significantly effective in preventing oral mucositis and is relevant in retaining epithelial integrity and mild inflammation of the lamina propria underlying the slightly eroded areas. Systemically, trans-caryophyllene modulated IFN- $\gamma$ levels at the establishment and remission stages of oral mucositis (Days 8 and 15 of the experiment, respectively), suggesting that this cytokine does not influence exacerbation (Day 11) of the ulcers directly. Other studies were performed using trans-caryophyllene, which focused on the immunomodulatory effects of the drug (49-51).

Wright et al (52) performed jejunal integrity studies in rats using the herbal extract-Iberogast- and concluded that this plant partially improved the histopathological features of 5-FU induced mucositis, but conferred no significant protection.

In 2014, Cheah et al (53) investigated the effects of grape seed extract in colon cancer and it demonstrated reduced severity of intestinal mucositis in patients undergoing 5-FU chemotherapy.

In addition, preventive treatment with atorvastatin displayed absence of epithelial dissolution with preserved integrity and mildly diffused inflammatory infiltrates. Systemically, atorvastatin negatively modulated the levels of TNF- $\alpha$ and INF- $\gamma$ during the development of mucositis at all stages (Days 8,11 and 15).

Serological analysis showed that trans-caryophyllene significantly reduces IFN- $\gamma$ levels, whereas atorvastatin reduces TNF- $\alpha$ levels. In addition to these results, there was a significant inhibition of leukopenia, as per previously published data (25).

Histologically, oral wounds showed that prevention with trans-caryophyllene was effective and developed no ulcers, whereas atorvastatin was associated with better surface recovery of the ulcerative wounds. Together, these results suggest that trans-caryophyllene prevents oral mucositis and reduces the systemic production of pro-inflammatory mediators.

Finally, we highlight the importance of exploring drugs to establish preventive protocols for oral mucositis that are secondary to chemotherapy and radiotherapy, since only symptomatic treatment is currently recommended, and only includes painkillers associated with nutritional support (46).

\section{Acknowledgements}

The authors would like to thank Professor Akinori Cardozo Nagato (Department of Physiology, Federal University of Juiz de Fora, Brazil) for statistical analysis.

\section{Funding}

This work received support of Rede Mineira de Bioterismo/ FAPEMIG (Minas Gerais State Research Support Foundation) number 31/11 and TOXIFAR (Minas Gerais Network of Toxicological and Pharmacological Tests of Therapeutic Products) number 26/11; Post Graduation Program at the Federal University of Juiz de Fora.

\section{Availability of data and materials}

All data generated or analyzed during this study are included in this published article.

\section{Authors' contributions}

All authors participated in the design, interpretation of the experiments, analysis of the data, and review of the manuscript. 
MIDCC, CNC, BJVA and FMA designed the experiments. MIDCC, BJVA and FMA performed pathologic anatomy. MIDCC and JOAC performed the immunohistochemistry experiments. MIDCC and JOAC were involved in ELISA. MIDCC, CNC, BJVA and FMA wrote the text. All authors read and approved the final manuscript.

\section{Ethics approval and consent to participate}

The experimental research was carried out on rodents (Rattus novergicus) having been approved by the Animal Research Ethics Committee of the Federal University of Juiz de Fora under number 062/2011. All stages of the research were explained in the proper form and, after being verified by three evaluators, it was approved without changes or considerations.

\section{Patient consent for publication}

Not applicable.

\section{Competing interests}

The authors declare that they have no competing interests.

\section{References}

1. Kostler WJ, Hejna M, Wenzel C and Zielinski CC: Oral mucositis complicating chemotherapy and/or radiotherapy: Options for prevention and treatment. CA Cancer J Clin 51: 290-315, 2001.

2. Sonis ST: Mucositis as a biological process: A new hypothesis for the development of chemotherapy-induced stomatotoxicity. Oral Oncol 34: 39-43, 1998.

3. Sonis ST: New thoughts on the initiation of mucositis. Oral Dis 16: 597-600, 2010.

4. Sonis ST: The pathobiology of mucositis. Nat Rev Cancer 4: 277-284, 2004

5. Sonis ST and Costello KA: A database for mucositis induced by cancer chemotherapy. Eur J Cancer B Oral Oncol 31B: 258-260, 1995.

6. Stone R, Fliedner MC and Smiet AC: Management of oral mucositis in patients with cancer. Eur J Oncol Nurs 9 (Suppl 1) S24-S32, 2005.

7. Trotti A, Bellm LA, Epstein JB, Frame D, Fuchs HJ, Gwede CK, Komaroff E, Nalysnyk L and Zilberberg MD: Mucositis incidence, severity and associated outcomes in patients with head and neck cancer receiving radiotherapy with or without chemotherapy: A systematic literature review. Radiother Oncol 66: 253-262, 2003

8. Trucci VM, Veeck EB and Morosolli ARC: Current strategies for the management of oral mucositis induced by radiotherapy or chemotherapy. Rev Odonto Cienc 24: 309-314, 2009.

9. Lalla RV, Sonis ST and Peterson DE: Management of oral mucositis in patients who have cancer. Dent Clin North Am 52: 61-77, 2008.

10. Peterson DE: New strategies for management of oral mucositis in cancer patients. J Support Oncol 4 (Suppl 1): S9-S13, 2006.

11. Yeoh AS, Gibson RJ, Yeoh EE, Bowen JM, Stringer AM, Giam KA and Keefe DM: A novel animal model to investigate fractionated radiotherapy-induced alimentary mucositis: The role of apoptosis, p53, nuclear factor-kappaB, COX-1, and COX-2. Mol Cancer Ther 6: 2319-2327, 2007.

12. Parkin J and Cohen B: An overview of the immune system. Lancet 357: 1777-1789, 2001.

13. Goldman L and Ausiello D (eds): Principles of cancer treatment In: Internal Medicine Treaty. Campus Elsevier, Rio de Janeiro, 2005 .

14. Kolios G, Petoumenos C and Nakos A: Mediators of inflammation: Production and implication in inflammatory bowel disease. Hepatogastroenterology 45: 1601-1609, 1998

15. Mitchell R, Kumar V, Abbas A and Fausto N: Robbins and Cotran Pathologic Basis of Diseases. 7th edition. Elsevier, Rio de Janeiro, 2006.
16. Aarestrup B: In situ evaluation of mediators of the inflammatory response and apoptosis process in chronic periodontitis in patients with AIDS. Niteroi, Universidade Federal Fluminense, 2006.

17. Baliga RS, Chaves AA, Jing L, Ayers LW and Bauer JA: AIDS-related vasculopathy: Evidence for oxidative and inflammatory pathways in murine and human AIDS. Am J Physiol Heart Circ Physiol 289: H1373-H1380, 2005.

18. Duda A, Stange A, Lüftenegger D, Stanke N, Westphal D Pietschmann T, Eastman SW, Linial ML, Rethwilm A and Lindemann D: Prototype foamy virus envelope glycoprotein leader peptide processing is mediated by a Furin-like cellular protease, but cleavage is not essential for viral infectivity. J Virol 78: 13865-13870, 2004.

19. Consolaro A: Cellular stress: Precedes and is present in inflammation. In: Inflammation and Repair. Dental Press, Maringá, 2009.

20. Azevedo IM, Kumakura HS, Alloufa SL, Mourão TS, Souza PM, Carvalho MDF, Medeiros VB, Araújo-Filho I, Rêgo ACM and Medeiros AC: Effect of simvastatin in attenuation of mucositis induced by methotrexate in rats. J Surg Clin Res 1: 22-32, 2010.

21. Flores D and Lisart R: Effectiveness of palifermin in the prevention of oral mucositis in patients with haematological cancers. Farm Hosp 34: 163-169, 2010 (In Spanish).

22. Spielberger R, Stiff P, Bensinger W, Gentile T, Weisdorf D, Kewalramani T, Shea T, Yanovich S, Hansen K, Noga S, et al: Palifermin for oral mucositis after intensive therapy for hematologic cancers. N Engl J Med 351: 2590-2598, 2004.

23. Schmidt-Choudhury A, Furuta GT, Lavigne JA, Galli SJ and Wershil BK: The regulation of tumor necrosis factor-alpha production in murine mast cells: Pentoxifylline or dexamethasone inhibits IgE-dependent production of TNF-alpha by distinct mechanisms. Cell Immunol 171: 140-1446, 1996.

24. Raber-Durlacher JE, von Bültzingslöwen I, Logan RM, Bowen J, Al-Azri AR, Everaus H, Gerber E, Gomez JG, Pettersson BG, Soga Y, et al: Systematic review of cytokines and growth factors for the management of oral mucositis in cancer patients. Support Care Cancer 21: 343-355, 2013.

25. Campos MI, Vieira WD, Campos CN, Aarestrup FM and Aarestrup BJ: Atorvastatin and trans-caryophyllene for the prevention of leukopenia in an experimental chemotherapy model in Wistar rats. Mol Clin Oncol 3: 825-828, 2015.

26. Basile AC, Sertie JA, Freitas PC and Zanini AC: Anti-inflammatory activity of oleoresin from Brazilian Copaifera. J Ethnopharmacol 22: 101-109, 1988.

27. PereiraFJ,MartinsFT,CorrêaRS,MoreiraME,Costa A,SantosMH, Polo $\mathrm{M}$ and Barbosa LC: Isolation, chemical composition and anti-inflammatory activity of Copaifera langsdorffii Desf. fruit peels essential oil according to successive hydrodistillations. Acta Farm Bonaer 27: 369-374, 2008

28. Vilanova CM, Ribeiro SM, Machado RC, Vieira SM, Lima SG, Nunes PH and Martins MC: Evaluation of oil-resin activity of Copaifera sp. On gastric emptying in Rattus novergicus. Emir J Food Agricult 25: 394-397, 2013

29. Siddiqui MA and Wellington K: Palifermin: In myelotoxic therapy-induced oral mucositis. Drugs 65: 2139-2149, 2005.

30. Sonis ST: Efficacy of palifermin (keratinocyte growth factor-1) in the amelioration of oral mucositis. Core Evid 4: 199-205, 2009.

31. Fernandes ES, Passos GF, Medeiros R, da Cunha FM, Ferreira J, Campos MM, Pianowski LF and Calixto JB: Anti-inflammatory effects of compounds alpha-humulene and (-)-trans-caryophyllene isolated from the essential oil of Cordia verbenacea. Eur J Pharmacol 569: 228-236, 2007.

32. Leandro LM, Vargas Fde S, Barbosa PC, Neves JK, da Silva JA and da Veiga-Junior VF: Chemistry and biological activities of terpenoids from copaiba (Copaifera spp.) oleoresins. Molecules 17: 3866-3889, 2012.

33. Paiva LA, de Alencar Cunha KM, Santos FA, Gramosa NV, Silveira ER and Rao VS: Investigation on the wound healing activity of oleo-resin from Copaifera langsdorffi in rats. Phytother Res 16: 737-739, 2002

34. Ward A and Clissold SP: Pentoxifylline. A review of its pharmacodynamic and pharmacokinetic properties, and its therapeutic efficacy. Drugs 34: 50-97, 1987.

35. Sonis ST, Tracey C, Shklar G, Jenson J and Florine D: An animal model for mucositis induced by cancer chemotherapy. Oral Surg Oral Med Oral Pathol 69: 437-443, 1990.

36. Scully C, Epstein J and Sonis S: Oral mucositis: A challenging complication of radiotherapy, chemotherapy, and radiochemotherapy: Part 1, pathogenesis and prophylaxis of mucositis. Head Neck 25: 1057-1070, 2003. 
37. Cai Y, Wang Z, Li J, Li N, Wei F and Liu Q: Evaluation of an indirect ELISA using recombinant granule antigen Gra7 for serodiagnosis of Toxoplasma gondii infection in cats. J Parasitol 101: 37-40, 2015.

38. Grisham MB, Jourd'Heuil D and Wink DA: Nitric oxide. I Physiological chemistry of nitric oxide and its metabolites: Implications in inflammation. Am J Physiol 276: G315-G321, 1999.

39. Fekrazad R and Chiniforush N: Oral mucositis prevention and management by therapeutic laser in head and neck cancers. J Lasers Med Sci 5: 1-7, 2014.

40. Herrstedt J: Prevention and management of mucositis in patients with cancer. Int J Antimicrob Agents 16: 161-163, 2000.

41. Lara RN, da Guerra EN and de Melo NS: Macroscopic and microscopic effects of GaAIAs diode laser and dexamethasone therapies on oral mucositis induced by fluorouracil in rats. Oral Health Prev Dent 5: 63-71, 2007.

42. Pico JL, Avila-Garavito A and Naccache P: Mucositis: Its occurrence, consequences, and treatment in the oncology setting. Oncologist 3: 446-451, 1998.

43. Silverman S Jr: Diagnosis and management of oral mucositis J Support Oncol 5: 13-21, 2007.

44. Wardill HR, Bowen JM and Gibson RJ: New pharmacotherapy options for chemotherapy-induced alimentary mucositis. Expert Opin Biol Ther 14: 347-354, 2014.

45. Vanderhoof JA, Park JH, Mohammadpour H and Blackwood D: Effects of dietary lipids on recovery from mucosal injury. Gastroenterology 98: 1226-1231, 1990.

46. Lima V, Vidal FD, Rocha FA, Brito GA and Ribeiro RA: Effects of tumor necrosis factor-alpha inhibitors pentoxifylline and thalidomide on alveolar bone loss in short-term experimental periodontal disease in rats. J Periodontol 75: 162-168, 2004.

47. Jain P, Keservani R and Dahima R: In-vivo characterization of hydrogel for treatment of chemo-radiotherapy induced oral mucositis. Pharmacol Online 1: 1016-1025, 2010.
48. Allen R, Rapecki S and Higgs G: The role of IL-10 in the inhibition of LPS-mediated TNF release from human PBMCs by phosphodiesterase 4 (PDE4) inhibitors. Inflamm Res 46: 218 , 1997.

49. Dias DS, Fontes LB, Crotti AE, Aarestrup BJ, Aarestrup FM, da Silva Filho AA and Corrêa JO: Copaiba oil suppresses inflammatory cytokines in splenocytes of $\mathrm{C} 57 \mathrm{Bl} / 6$ mice induced with experimental autoimmune encephalomyelitis (EAE). Molecules 19: 12814-12826, 2014

50. Guo K, Mou X, Huang J, Xiong N and Li H: Trans-caryophyllene suppresses hypoxia-induced neuroinflammatory responses by inhibiting NF- $\kappa$ B activation in microglia. J Mol Neurosci 54: 41-48, 2014.

51. Veiga Junior VF, Rosas EC, Carvalho MV, Henriques MG and Pinto AC: Chemical composition and anti-inflammatory activity of copaiba oils from Copaifera cearensis Huber ex Ducke, Copaifera reticulata Ducke and Copaifera multijuga Hayne-a comparative study. J Ethnopharmacol 112: 248-254, 2007.

52. Wright TH, Yazbeck R, Lymn KA, Whitford EJ, Cheah KY, Butler RN, Feinle-Bisset C, Pilichiewicz AN, Mashtoub S and Howarth GS: The herbal extract, Iberogast, improves jejunal integrity in rats with 5-fluorouracil (5-FU)-induced mucositis. Cancer Biol Ther 8: 923-929, 2009.

53. Cheah KY, Howarth GS and Bastian SE: Grape seed extract dose-responsively decreases disease severity in a rat model of mucositis; concomitantly enhancing chemotherapeutic effectiveness in colon cancer cells. PLoS One 9: e85184, 2014.

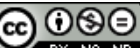

This work is licensed under a Creative Commons Attribution-NonCommercial-NoDerivatives 4.0 International (CC BY-NC-ND 4.0) License. 